\title{
Síndrome de Vog't-Koyanagi-Harada incompleta associada a HLA DRB1*01 em criança de quatro anos de idade: relato de caso
}

\author{
Incomplete Vogt-Koyanagi-Harada syndrome associated with HLA DRB1*01 in \\ a 4-year-old child:case report
}

\author{
Daniel Rocha Lucena ${ }^{1}$ \\ Jayter Silva Paula ${ }^{2}$ \\ Gleilton Carlos Mendonça Silva ${ }^{3}$ \\ Maria de Lourdes Veronese Rodrigues ${ }^{4}$
}

\begin{tabular}{|l|}
\hline RESUMO \\
\hline A síndrome de Vogt-Koyanagi-Haradaé doença crônica, sistêmica e auto- \\
imune, com manifestações oculares, nervosas, auditivas e tegumentares. \\
Descrevemos aqui o caso de uma criança com início dos sintomas aos \\
quatro anos e dois meses de idade, com positividade para o HLA \\
DRB1*01.
\end{tabular}

Descritores: Síndrome uveomeningoencefálica; Criança; Antígenos HLA-DR; Uveite/ etiologia; Uveite/diagnóstico; Relatos de casos [Tipo de publicação]
Trabalho realizado no Departamento de Oftalmologia, Otorrinolaringologia e Cirurgia de Cabeça e Pescoço Faculdade de Medicina da Universidade de São Paulo - USP - Ribeirão Preto (SP) - Brasil.

${ }^{1}$ Médico Assistente do Departamento de Oftalmologia, Otorrinolaringologia e Cirurgia de Cabeça e Pescoço Faculdade de Medicina da Universidade de São Paulo - USP - Ribeirão Preto (SP) - Brasil.

${ }^{2}$ Professor Colaborador do Departamento de Oftalmologia, Otorrinolaringologia e Cirurgia de Cabeça e Pescoço - Faculdade de Medicina da USP - Ribeirão Preto (SP) - Brasil.

3 Pós-graduando do Departamento de Oftalmologia, Otorrinolaringologia e Cirurgia de Cabeça e Pescoço - Faculdade de Medicina da USP - Ribeirão Preto (SP) - Brasil.

${ }^{4}$ Professora Associada do Departamento de Oftalmologia, Otorrinolaringologia e Cirurgia de Cabeça e Pescoço - Faculdade de Medicina da USP - Ribeirão Preto (SP) - Brasil.

Endereço para correspondência: Jayter Silva de Paula. Departamento de Oftalmologia, Otorrinolaringologia e Cirurgia de Cabeça e Pescoço, Hospital das Clínicas de Ribeirão Preto - Campus (USP). Av. Bandeirantes, 3900 - Ribeirão Preto (SP) CEP 14049-900

E-mail: jayterdepaula@yahoo.com.br

Recebido para publicação em 19.07.2005

Última versão recebida em 21.08.2006

Aprovação em 12.09.2006

Nota Editorial: Depois de concluída a análise do artigo sob sigilo editorial e com a anuência da Dra. Luciana Peixoto Finamor sobre a divulgação de seu nome como revisora, agradecemos sua participação neste processo.

\section{INTRODUÇÃO}

A síndrome de Vogt-Koyanagi-Harada (SVKH) é uma doença sistêmica que envolve tecidos que contém melanina. É caracterizada por uma panuveíte bilateral, crônica, granulomatosa associada a manifestações variáveis de comprometimento neurológico, auditivo e cutâneo. O envolvimento ocular bilateral é necessário para caracterizar o diagnóstico, necessariamente sem história prévia de cirurgia ou trauma ocular. Os sinais mais comuns incluem iridociclite, vitreíte, edema ou hiperemia de disco óptico, espessamento coroideano e descolamento de retina neuro-sensorial ${ }^{(1-2)}$.

Os critérios diagnósticos, atualmente utilizados, foram publicados a partir do I "Workshop" Internacional da SVKH e incluem: (1) ausência de história prévia de trauma ou cirurgia ocular; (2) ausência de evidência de doença ocular concomitante; (3) envolvimento ocular bilateral precoce (com áreas focais de fluido sub-retiniano ou descolamento seroso de retina) ou tardio (despigmentação, fundo de olho em pôr-do-sol, cicatrizes coriorretinianas despigmentadas de Dalen-Fuchs, e migração ou acúmulo de epitélio pigmentar da retina); (4) história ou sinais auditivos e/ou neurológicos; (5) alterações cutâneas que apareçam durante ou após as manifestações neurológicas e oculares ${ }^{(3)}$.

Baseado nesses critérios, a SVKH pode ser classificada em completa, quando todos os critérios são preenchidos, incompleta, quando estão presentes os critérios $1,2,3$ e 4 ou 5, ou provável, quando apenas os critérios 1,2 e 3 são preenchidos ${ }^{(3)}$. A idade usual de início dos sintomas é entre 20 e 50 anos, sendo que apenas $5 \%$ dos casos foram descritos com idade inferior aos $16 \operatorname{anos}^{(4)}$.

Este artigo descreve um caso raro de SVKH incompleta complicada com neovascularização sub-retiniana bilateral e associada à expressão do HLA DRB $1 * 01$ em uma criança com 4 anos e 2 meses de idade, o qual deve ser o mais jovem caso relatado com esta síndrome. 


\section{RELATO DE CASO}

Criança branca, sexo masculino, foi admitida no Ambulatório de Oftalmologia do Hospital das Clínicas da Faculdade de Medicina de Ribeirão Preto - Universidade de São Paulo, com história de hiperemia, dor ocular e fotofobia em ambos os olhos (AO) há 4 meses. Queixava-se ainda de cefaléia freqüente, dislalia e possível hipoacusia no período.

Ao exame oftalmológico apresentava boa fixação em ambos olhos, porém o paciente não informava acuidade visual, por não entender optotipos. À biomicroscopia evidenciou-se intensa reação em câmara anterior, incluindo "Tyndall" celular de 3+/4+ e finos precipitados ceráticos. Havia também extensas sinéquias posteriores em AO. Ao exame fundoscópico o corpo vítreo apresentava-se sem "haze", e lesões amareladas compatíveis com áreas de coroidite podiam ser observadas inferiormente no pólo posterior e na região nasal AO. Optouse pelo tratamento tópico com colírios de dexametasona $1 \%$ e atropina $1 \%$, até a conclusão dos exames complementares solicitados.

Exames de rotina e hemograma estavam dentro dos limites da normalidade, assim como a pesquisa de anticorpos antinúcleo, anti-cardiolipina, fator reumatóide, componentes do complemento sérico, RIF-toxoplasmose, VDRL, FTA-ABS, enzima conversora de angiotensina e raios- $\mathrm{x}$ de tórax. A velocidade de hemossedimentação era de $5 \mathrm{~mm} / 1$ hora.

No retorno em 2 meses, a angiofluoresceinografia evidenciou alguns pontos de vazamento no epitélio pigmentar da retina ("pin-points"), com acúmulo de contraste no espaço sub-retiniano em AO (figura não disponível, de baixa qualidade, dada pela não colaboração do paciente). O exame audiométrico evidenciou perda auditiva leve a partir de $6 \mathrm{kHz}$, bilateralmente. Concluiu-se o diagnóstico de síndrome de Vogt-Koyanagi-Harada incompleta e se instituiu o seguinte tratamento: albendazol $400 \mathrm{mg} /$ dia por 3 dias (profilático), colírios de dexametasona $1 \%$ e atropina $1 \%$, e prednisona $1 \mathrm{mg} / \mathrm{kg} / \mathrm{dia}$ (via oral). Após 1 mês, a acuidade visual corrigida era 1,0 (paciente informando adequadamente os optotipos), com controle da inflamação e ausência de coroidite ativa em AO.

Apesar da retirada gradual do corticóide sistêmico, houve recorrência da doença após 7 meses de seguimento, com recrudescimento da baixa acuidade visual $(0,2$ no olho direito, e 0,4 no olho esquerdo) e da uveíte anterior AO. Optou-se novamente pelo tratamento com a dose inicial de corticosteróide sistêmico, associado a metotrexate 7,5 mg/semana (esquema indicado pelo setor de imunopediatria). Apesar deste esquema de tratamento, observou-se o aparecimento de uma membrana neovascular sub-retiniana na região macular de AO. Consequientemente, indicou-se ciclosporina-A (50 mg de $12 / 12$ horas), com a suspensão abrupta do metotrexate e progressiva da corticoterapia oral.

Após 3 meses de tratamento, a fundoscopia revelava a presença de tecido sub-retiniano de aspecto gliótico compatível com cicatriz disciforme, rarefação generalizada de epitélio pigmentar da retina, cicatrizes do tipo Dalen-Fuchs e fundo de olho em pôr-do-sol bilateralmente (Figura 1). A tipificação dos alelos de histocompatibilidade (HLA) evidenciou os subtipos DRB $1 * 01, \mathrm{DRB} 1 * 14$, DRB3 e DQB $1 * 05$.

Atualmente o paciente se apresenta assintomático, em uso de ciclosporina A. Não há atividade inflamatória intra-ocular, porém mantém acuidade visual corrigida de 0,2 e lesões cicatriciais acometendo a região macular AO. Acrescenta-se ainda que, em nenhum momento da evolução clínica, o paciente apresentou sinais ou sintomas de artrite.

\section{DISCUSSÃO}

A SVKH é mais freqüente entre negros, orientais, índios americanos e hispânicos ${ }^{(5)}$. Os principais diagnósticos diferenciais desta síndrome incluem: esclerites posteriores, oftalmia simpática, coroidite multifocal, dentre outras ${ }^{(6)}$. Sua etiolo-
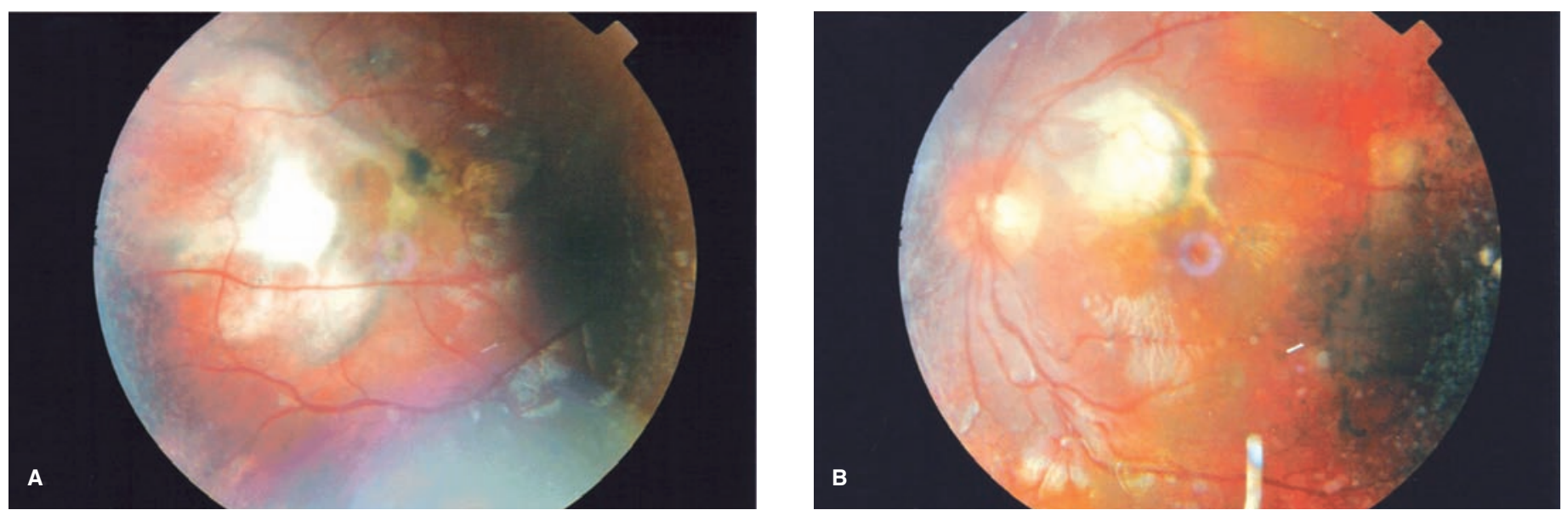

Figura 1 - Retinografia do paciente com síndrome de Vogt-Koyanagi-Harada, fase convalescente. A) Olho direito: tecido gliótico sub-retiniano acometendo pólo posterior, configurando cicatriz disciforme secundária a membrana neovascular sub-retiniana. B) Olho esquerdo: cicatriz disciforme justafoveolar superior e fundo de olho em "pôr-do-sol". Notar também áreas de acúmulo de pigmento em ambos os olhos. 
gia é obscura, sendo que a predileção por raças pode condizer com uma predisposição genética ${ }^{(5)}$. Há indícios de que uma infecção, provavelmente viral, nestes pacientes poderia induzir a formação de novos antígenos na superfície dos melanócitos, os quais desencadeariam uma reação imune antígenoespecífica $^{(7)}$.

No caso relatado, o paciente apresentou um quadro de uveíte difusa, com iridociclite altamente sintomática e descolamento seroso da retina bilateralmente. Houve, posteriormente, recorrência da inflamação em segmento anterior e formação de membrana neovascular sub-retiniana AO. Também apresentou hipoacusia documentada, que associada aos achados citados acima, confirma a hipótese de SVKH incompleta.

Há relatos na literatura do diagnóstico de SVKH em crianças aos $6^{(8)}$ e $7^{(9)}$ anos de idade. $O$ caso mais jovem publicado até o momento é o de uma criança de 4 anos e 6 meses de idade por ocasião do início dos sintomas ${ }^{(10)}$. No presente caso, a criança apresentava 4 anos e 2 meses, o mais precoce dentre aqueles revisados na literatura. Embora haja disparidade nos achados entre os casos citados, todos têm em comum o fato de não preencherem os 5 critérios para a SVKH completa segundo a classificação atualmente aceita ${ }^{(3)}$. Além disso, esses trabalhos foram publicados anteriormente à revisão de alguns desses critérios.

Parece haver associação da SVKH com os subtipos dos alelos de histocompatibilidade HLA-DRB1 e-DQB1 $1^{(11-15)}$. Dentre os subtipos apresentados pelo paciente, os alelos HLADRB $1 * 01$ e - DQB $1 * 05$, os quais apresentam baixa prevalência na população local ${ }^{(16)}$, já foram previamente associados de forma direta a essa doença ${ }^{(15)}$.

Algumas outras doenças auto-imunes também se associam a alguns subtipos dos alelos DRB $1 * 01$, tais como a artrite reumatóide juvenil ${ }^{(17)}$. No entanto, muitos deles, incluindo também o DRB 1*14, se apresentam como fatores ditos "de proteção" de algumas formas dessa doença ${ }^{(18-20)}$.

A presença da associação de expressão dos alelos DRB1*01 e DQB $1 * 05$, juntamente com a apresentação clínica de inflamação do segmento anterior altamente sintomática e achados típicos de segmento posterior, fortalece a hipótese diagnóstica levantada, afastando outras possíveis na infância ${ }^{(15)}$.

Embora muito incomum em crianças ${ }^{(6,8-10)}$, a SVKH deve ser considerada no diagnóstico diferencial de quadros de panuveíte idiopática com resposta terapêutica rápida com corticoterapia sistêmica. Nesses casos, a família do paciente deve ser informada quanto ao curso da doença e seu possível prognóstico visual reservado.

\section{ABSTRACT}

Vogt-Koyanagi-Harada syndrome is chronic systemic autoimmune disease with ocular, nervous, auditory and tegumental manifestations. We report here the case of a child with onset of symptoms at four years and two months of age, with positive HLA DRB $1 * 01$.

Keywords: Uveomeningoencephalitic syndrome; Child; HLADR antigens; Uveitis/etiology; Uveitis /diagnosis; Case reports [Publication type]

\section{REFERÊNCIAS}

1. Touitou V, Escande C, Boddaghi B, Cassoux N, Wechsler B, Lemaitre C, et al. [Diagnostic and therapeutic management of Vogt-Koyanagi-Harada syndrome]. J Fr Ophtalmol. 2005;28(1):9-16. French.

2. Bezerra HL, Santos LP, Carvalho AM, Muccioli C, Belfort Junior R. Síndrome de Vogt-Koyanagi-Harada: revisão de 89 casos. Arq Bras Oftalmol. 1998; 61(3):331-4.

3. Read RW, Holland GN, Rao NA, Tabbara KF, Ohno S, Arellanes-Garcia L, et al. Revised diagnostic criteria for Vogt-Koyanagi-Harada disease: report of an international committee on nomenclature. Am J Ophthalmol. 2001;131(5): 647-52.

4. Laghmari M, Karim A, Ibrahimy W, Essakalli NH, Mohcine Z. [Vogt-KoyanagiHarada syndrome in children]. J Fr Ophtalmol. 2002;25(6):636-40. French.

5. Beniz J, Forster DJ, Lean JS, Smith RE, Rao NA. Variations in clinical features of the Vogt-Koyanagi-Harada syndrome. Retina. 1991;11(3):275-80.

6. Moorthy RS, Inomata H, Rao NA. Vogt-Koyanagi-Harada syndrome. Surv Ophthalmol. 1995;39(4):265-92.

7. Matsuda H, Sugiura S. Ultrastructural changes of the melanocyte in VogtKoyanagi-Harada syndrome and sympathetic ophthalmia. Jpn J Ophthalmol. 1971;15:69-80.

8. Perkins ES. Pattern of uveitis in children. Br J Ophthalmol. 1966;50(4):169-85.

9. Forster DJ, Green RL, Rao NA. Unilateral manifestation of the Vogt-KoyanagiHarada syndrome in a 7-year-old child. Am J Ophthalmol. 1991;111(3):380-2.

10. Gruich MJ, Evans OB, Storey JM, Bradley ST, Chen CJ. Vogt-KoyanagiHarada syndrome in a 4-year-old child. Pediatr Neurol. 1995;13(1):50-1.

11. Zhang M, Oiu C, Hu T. [Association of HLA-DRB genes with Vogt-Koyanagi-Harada syndrome in a Chinese Han population]. Zhongguo YI Xue Ke Xue Yuan Xue Bao. 2000;22(1):36-40. Chinese.

12. Goldberg AC, Yamamoto JH, Chiarella JM, Marin ML, Sibinelli M, Neufeld $\mathrm{R}$, et al. HLA-DRB1*0405 is the predominant allele in Brazilian patients with Vogt-Koyanagi-Harada disease. Hum Immunol. 1998;59(3):183-8.

13. Weisz JM, Holland GN, Roer LN, Park MS, Yuge AJ, Moorthy RS, et al. Association between Vogt-Koyanagi-Harada syndrome and HLA-DR1 and -DR4 in Hispanic patients living in southern California. Ophthalmology. 1995; 102(7):1012-5.

14. Arellanes-Garcia L, Bautista N, Mora P, Ortega-Larrocea G, Burguet A, Gorodezky C. HLA-DR is strongly associated with Vogt-Koyanagi-Harada disease in Mexican Mestizo patients. Ocul Immunol Inflamm. 1998;6(2):93-100.

15. Levinson RD, See RF, Rajalingam R, Reed EF, Park MS, Rao NA, Holland GN. HLA-DRB1 and -DQB1 alleles in mestizo patients with Vogt-KoyanagiHarada's disease in Southern California. Hum Immunol. 2004;65(12):1477-82.

16. Louzada-Junior P, Smith AG, Hansen JA, Donadi EA. HLA-DRB1 and DQB1 alleles in the Brazilian population of the northeastern region of the state of Sao Paulo. Tissue Antigens. 2001;57(2):158-62.

17. Miterski B, Drynda S, Boschow G, Klein W, Oppermann J, Kekow J, Epplen JT. Complex genetic predisposition in adult and juvenile rheumatoid arthritis. BMC Genet. 2004;5:2.

18. Malagon C, Van Kerckhove C, Giannini EH, Taylor J, Lovell DJ, Levinson JE, et al. The iridocyclitis of early onset pauciarticular juvenile rheumatoid arthritis: outcome in immunogenetically characterized patients. J Rheumatol. 1992;19(1):160-3.

19. Haas JP, Truckenbrodt H, Paul C, Hoza J, Scholz S, Albert ED. Subtypes of HLA-DRB $1 * 03, * 08, * 11, * 12, * 13$ and $* 14$ in early onset pauciarticular juvenile chronic arthritis (EOPA) with and without iridocyclitis. Clin Exp Rheumatol. 1994;12 Suppl 10:S7-14.

20. Garavito G, Yunis EJ, Egea E, Ramirez LA, Malagon C, Iglesias A, et al. HLA-DRB1 alleles and HLA-DRB1 shared epitopes are markers for juvenile rheumatoid arthritis subgroups in Colombian mestizos. Hum Immunol. 2004;65(4):359-65. 